\title{
Locally Advanced Colon Cancer: Evaluation of Current Clinical Practice and Treatment Outcomes at the Population Level
}

\author{
Charlotte E.L. Klaver, MDa; Lieke Gietelink, MD ${ }^{\mathrm{b}, \mathrm{c}}$; Willem A. Bemelman, MDa; \\ Michel W.J.M. Wouters, MD, ; Theo Wiggers, MD' ; Rob A.E.M. Tollenaar, MD ${ }^{\mathrm{b}, \mathrm{c}}$; and \\ Pieter J. Tanis, MD, $\mathrm{PhD}^{\mathrm{a}}$; on behalf of the Dutch Surgical Colorectal Audit Group
}

\begin{abstract}
Background: The goal of this study was to evaluate current clinical practice and treatment outcomes regarding locally advanced colon cancer (LACC) at the population level. Methods: Data were used from the Dutch Surgical Colorectal Audit from 2009 to 2014. A total of 34,527 patients underwent resection for non-LACC and 6,918 for LACC, which was defined as CT4 and/or pT4 stage. LACC was divided into those with multivisceral resection (LACC-MV; $n=3,385$ ) and without (LACC-noMV; $n=1,595)$. Guideline adherence, treatment strategy, and short-term outcomes were evaluated. Results: Guideline adherence was $>90 \%$ regarding preoperative imaging and $\geq 80 \%$ regarding preoperative multidisciplinary team (MDT) discussion. In the elective setting, neoadjuvant chemoradiotherapy (chemoRT) was applied in $6.2 \%$ of the CT4 cases, and neoadjuvant chemotherapy in 4.0\%. R0 resection rates were $99 \%, 91 \%$, and $87 \%$ in patients with non-LACC, LACCnoMV, and LACC-MV, respectively $(P<.001)$. A postoperative complicated course occurred in $17 \%, 25 \%$, and $29 \%$ of patients $(P<.001)$, and the 30-day/in-hospital mortality rate was 3.6\%,6.0\%, and $5.4 \%(P<.001)$ in the non-LACC, LACC-noMV, and LACC-MV groups, respectively. Discussion/Conclusions: This population-based study suggests that there is room for improvement in the treatment of LACC, with regard to short-term surgical outcomes and oncologic outcomes (ie, radicality of resection). Improvement might be expected from optimized preoperative imaging, routine MDT discussions, and further specialization and centralization of care. Optimized use of neoadjuvant treatment strategies based on already available and upcoming evidence is likely to result in a better margin status and thereby a better long-term prognosis. Furthermore, lower R0 resection rates in an emergency setting suggest a potential role for bridging strategies in order to enable optimal staging, neoadjuvant treatment, and elective surgery by a surgical team most optimally qualified for the procedure.
\end{abstract}

\section{Background}

Colon cancer is highly prevalent worldwide ${ }^{1}$ and a major public health problem. A substantial group of patients $(10 \%-15 \%)$ presents with locally advanced colon cancer (LACC), which has an important impact on disease management and prognosis. Standard curative-intent treatment of LACC is a complete resection of the tumor (RO resection) $)^{2,3}$ followed by

From the aDepartment of Surgery, Academic Medical Centre, University of Amsterdam, Amsterdam; 'bepartment of Surgery, Leiden University Medical Centre, Leiden; 'Dutch Institute for Clinical Auditing, Leiden; dDepartment of Surgical Oncology, the Netherlands Cancer Institute/Antoni van Leeuwenhoek Hospital, Amsterdam; and eDepartment of Surgical Oncology, University Medical Center Groningen, Groningen, the Netherlands.

Submitted February 8, 2016; accepted for publication October 6, 2016.

The authors have disclosed that they have no financial interests, arrangements, adjuvant systemic chemotherapy, depending on the age and clinical condition of the patient.

LACC can be subdivided into T4a stage with serosal ingrowth and $\mathrm{T} 4 \mathrm{~b}$ stage with ingrowth into nearby tissues or organs. ${ }^{4}$ In order to achieve a RO resection of T4b tumors, the surgical approach should include a multivisceral resection with or without neoadjuvant downstaging. ${ }^{5,6}$ Despite the prevalence of LACC and its

affiliations, or commercial interests with the manufacturers of any products discussed in this article or their competitors.

Contributions: Study conception and design: Klaver, Gietelink, Tanis. Analysis and interpretation of data: Klaver, Gietelink, Tanis. Manuscript writing: Klaver, Gietelink, Tanis. Critical manuscript revision: Bemelman, Wouters, Wiggers, Tollenaar, Tanis. All authors approved the final version of the manuscript. Correspondence: Pieter J. Tanis, MD, PhD, Academic Medical Centre, University of Amsterdam, Department of Surgery, Meibergdreef 9, 1105 AZ Amsterdam, the Netherlands. E-mail: p.j.tanis@amc.nl 
Klaver et al

relatively poor prognosis, treatment of LACC is still an underexposed area in the field of colorectal cancer (CRC) care when compared with, for example, the extensive literature on locally advanced rectal cancer.

The Dutch Surgical Colorectal Audit (DSCA) has been evaluating and reporting on the quality of care of primary CRC surgery since 2009. 7,8 The goal of this study was to evaluate current clinical practice regarding and short-term outcomes of the treatment of LACC at the population level using DSCA data.

\section{Methods}

\section{Data Set}

Data were derived from the DSCA, a disease-specific national audit. The audit collects information on patients, tumors, treatment characteristics, and outcomes and contains data from approximately 97\% of all patients who underwent resection for primary CRC in the Netherlands. Data entry is obligatory and data are stored in a highly secured online database. All 92 Dutch hospitals participate and appoint a surgeon responsible for data entry. The data set is cross-checked several times with data registered in the Netherlands Cancer Registry to ensure completeness. Detailed information on the validity, collection, and methodology of the data set was published previously. ${ }^{7,8}$

\section{Patients}

For this study, no ethical approval or informed consent was required under Dutch law. All patients who underwent surgery between January 1, 2009, and December 31, 2014, and were registered before March 15, 2015, were evaluated. Patients with multiple synchronous tumors within the colon were included, but patients with a second tumor in the rectum were excluded. Patients were considered eligible for this study if at least the following data were available: tumor location, date of surgery, and survival status at the time of hospital discharge. Based on these criteria, $98.7 \%(n=39,491)$ of all registered patients were available for analysis. Furthermore, for the purpose of the present analysis, all patients with metastatic disease were excluded.

\section{Definitions}

In the DSCA, both clinical and pathologic $\mathrm{T}$ stage were available, but without subdivision in $\mathrm{T} 4 \mathrm{a}$ and T4b. LACC was defined as all patients with a registered clinical and/or pathologic T4 stage. The extent of surgery for the primary tumor was registered in the DSCA as no, limited, or extensive additional resections for local ingrowth. Limited additional resections were defined as resections of the abdominal wall, omentum, or ovaries. Extensive additional resections referred to resections of the pancreas, spleen, kidney, liver, stomach, bladder, ureters, or uterus, or additional bowel resections. The organs involved or the exact locations of the additional resections are not specified. The variable additional resections for local ingrowth were used to define 2 subgroups: LACC without additional/ multivisceral resections (LACC-noMV) and LACC with limited or extended additional/multivisceral resections (LACC-MV). All other colon cancer resections were referred to as non-LACC. In short, the following 3 subgroups were used in this study: LACC-noMV, which comprised patients who underwent a resection of a cT4 and/or pT4 colon carcinoma without the need for a multivisceral resection; LACC-MV, which comprised patients who underwent a multivisceral resection of a cT4 and/or pT4 colon carcinoma; and non-LACC, which comprised patients who underwent a resection for a T1-3 colon cancer (ie, a tumor not classified as either cT4 or pT4).

Emergency surgery was defined as surgery performed within 12 hours after the procedure was scheduled. Urgent surgery referred to semiurgent procedures that were scheduled $>12$ hours before being performed but outside of the elective program. The surgical approach was either open, laparoscopic, or converted laparoscopic surgery. Hospital volume was defined as the number of resections performed for LACC-MV per hospital per year.

Outcome variables were guideline adherence (guidelines are provided later), radicality of resection, and postoperative course. The subcategories for radicality of resections were $\mathrm{RO}$, which denoted complete tumor resection with all margins histologically uninvolved; R1, which denoted incomplete resection with microscopic surgical resection margin involvement; and R2, which denoted incomplete tumor resection with gross residual tumor that was not resected. A complicated course referred to a postoperative complication leading to a reintervention, hospital stay $>14$ days, or 
death. Surgical complications were directly related to the surgical procedure (ie, anastomotic leakage, abscess, bleeding, ileus). Nonsurgical complications were not directly related to the surgery (ie, postoperative pneumonia). Mortality was defined as 30-day or in-hospital mortality.

\section{Treatment for LACC According to the Dutch Guidelines}

The Dutch CRC guideline, which was used until June 2014, advised to routinely perform a preoperative CT scan for colon cancer. In the case of LACC, this was aimed at optimizing the surgical approach with en bloc multivisceral resection and at considering neoadjuvant therapy. Preoperative chemoradiotherapy (chemoRT) had to be considered if $\mathrm{RO}$ resection was found to be unachievable based on CT imaging or intraoperative findings from an explorative laparotomy. Postoperative chemoRT had to be considered in cases of $\mathrm{R} 2$ resection with clipping of the operative field. In the revised guideline available April 2014 (www.oncoline.nl/colorectaalcarcinoom), preoperative imaging and multidisciplinary team (MDT) discussion were recommended in order to select the optimal treatment strategy. Preoperative systemic therapy is added as a neoadjuvant treatment option, besides chemoRT. Postoperative chemoRT for LACC is no longer advised (see supplemental eAppendix 1, available with this article at INCCN.org.).

\section{Statistical Analysis}

Differences in baseline characteristics and outcome variables between patients with non-LACC, LACC-noMV, and LACC-MV were analyzed using a chi-square test or Fisher exact test in the case of categorical variables. The Kruskal-Wallis oneway analysis of variance was used for continuous (nonparametric) variables. R0 resection proportions were compared between different subgroups based on resection type, surgical approach, neoadjuvant treatment, and hospital volume. To determine potential improvement in quality of care over time, outcome parameters were plotted against year of registration. The trend over time was analyzed using the chi-square test for linearity. $P<.05$ was considered statistically significant. Statistical analyses were performed in PASW Statistics, version 22 (PASW Inc., Chicago, IL).

\section{Results}

\section{Patients}

Of all patients with colon cancer registered between January 1, 2009, and December 31, 2014, in 92 Dutch hospitals, 39,491 were eligible for analysis. A total of 4,964 patients were staged as M1 and excluded from this analysis. Clinical T stage was known in only $27 \%$ of the remaining 34,527 patients, and cT4 stage was registered in 578 patients. A total of 4,730 patients had a pathologic T4 tumor. There was an overlap between these groups in the case of 328 patients who had tumors classified as both cT4 and pT4, which resulted in a total of 4,980 patients with cT4 and/ or pT4 stage disease (LACC); the remaining 29,547 $(86 \%)$ were patients classified as non-LACC (Figure $1)$. In the LACC group, 3,385 patients $(68 \%)$ were classified as LACC-noMV and 1,595 patients (32\%) as LACC-MV. Limited and extensive additional resections were performed in $53 \%$ and $47 \%$ of the LACC-MV patients, respectively.

\section{Baseline Characteristics and Surgery}

Baseline characteristics of the 3 subgroups are outlined in Table 1. Compared with non-LACC patients, those with LACC-noMV and those with LACC-MV experienced more preoperative tumor complications ( $34 \%$ vs $51 \%$ and $52 \%$, respectively). The percentage of procedures in the emergency/ urgent setting was $14 \%$ for non-LACC and $33 \%$ and

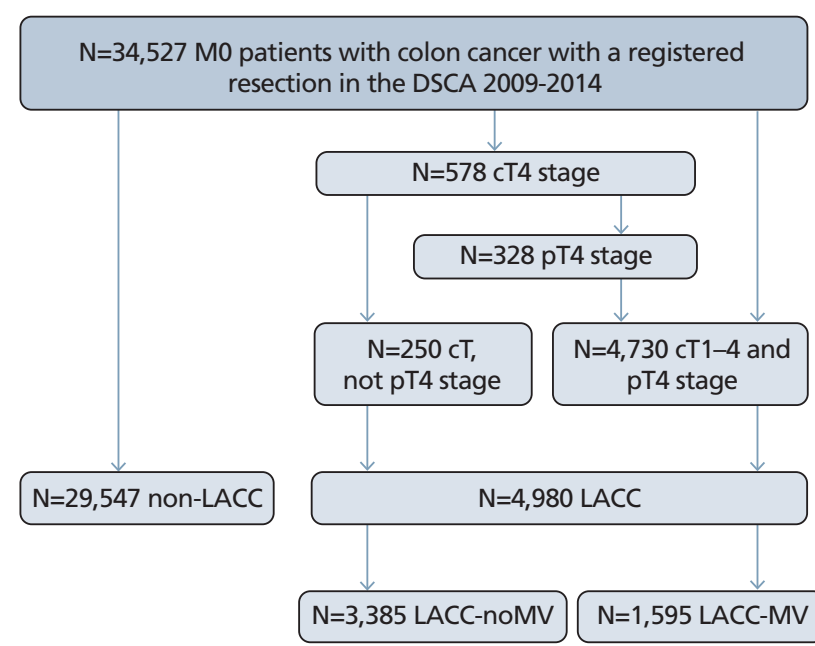

Figure 1. Subdivision of patients into non-LACC, LACC-noMV and LACC-MV.

Abbreviations: DSCA, Dutch Surgical Colorectal Audit; LACC, locally advanced colon cancer; MV, multivisceral resection required; noMV, no multivisceral resection required; non-LACC, all other colon cancer resections. 
Klaver et al

Table 1. Baseline Characteristics

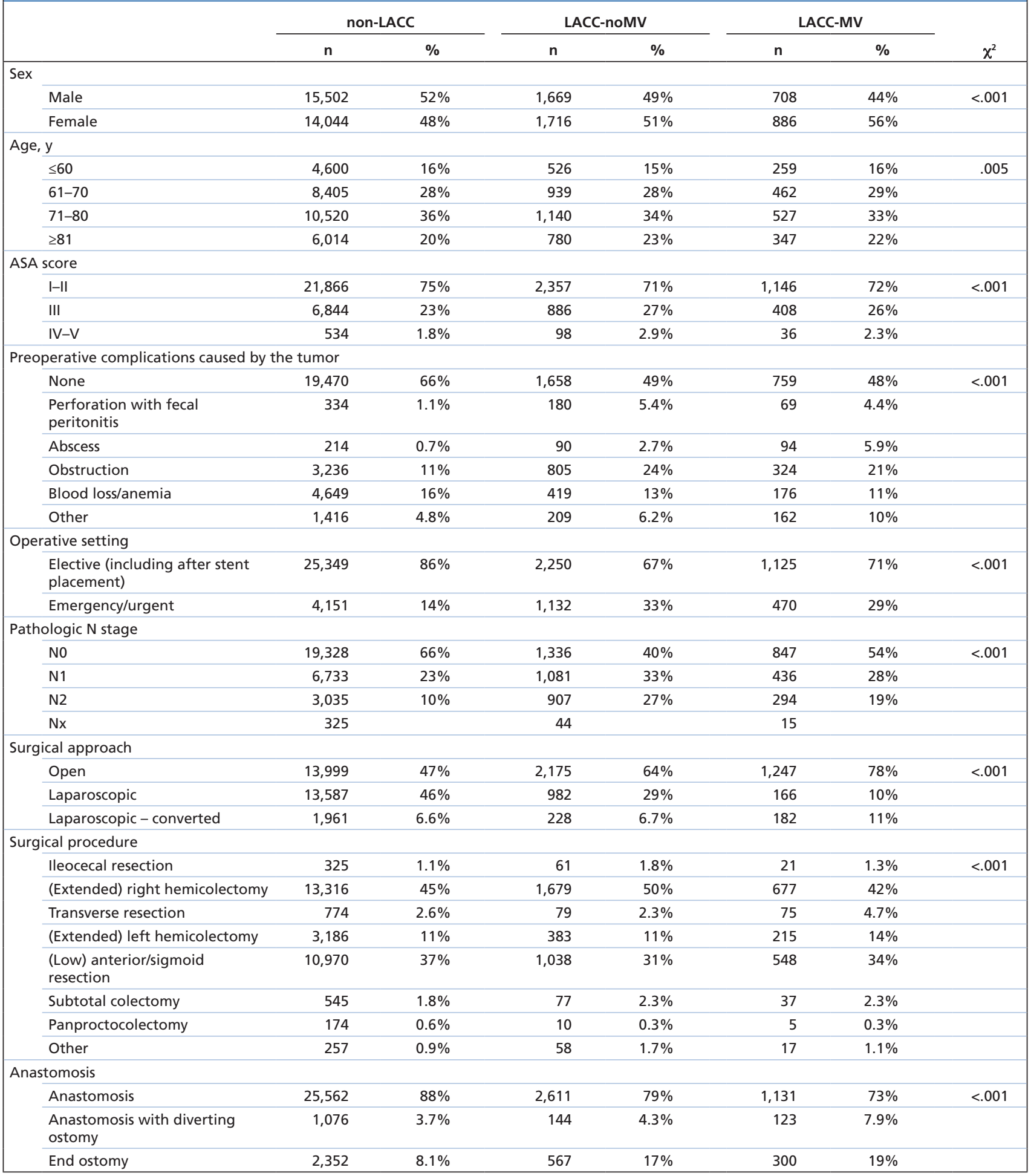

Abbreviations: ASA, American Society of Anesthesiologists; LACC, locally advanced colon cancer; MV, multivisceral resection required; noMV, no multivisceral resection required. 
29\% for LACC-noMV and LACC-MV patients, respectively. LACC was associated with a higher proportion of nodal positivity compared with nonLACC. Within the LACC group, nodal positivity was higher for LACC-noMV compared with LACCMV (60\% vs $47 \%)$.

The surgical procedure commenced laparoscopically in $53 \%$ of patients with non-LACC, in $36 \%$ of those with LACC-noMV, and in 21\% of those with LACC-MV. Conversion rates were $13 \%, 19 \%$, and $52 \%$, respectively. The proportion of primary anastomoses was considerably lower in LACC-MV patients compared with LACC-noMV and non-LACC patients (Table 1).

\section{Guideline Adherence}

Preoperatively, an abdominal CT at the least was performed in $92 \%$ of patients with LACC-noMV and in $95 \%$ of those with LACC-MV (Table 2); percentages were slightly higher (94\% and 96\%, respectively) if emergency/urgent procedures are excluded. Regarding preoperative MDT discussion, $80 \%$ of LACC-noMV and $82 \%$ of LACC-MV patients undergoing elective surgery were discussed during an MDT meeting. Considering cT4 stage in the elective setting only, 6.2\% $(n=22)$ of patients with LACC (either no-MV or MV) received neoadjuvant chemoRT and $4.0 \%(n=14)$ neoadjuvant systemic therapy.

\section{Outcome Variables}

Compared with non-LACC, the overall R0 resection proportion was lower in patients with LACC (99\% vs 90\%, respectively) (Table 3). A higher proportion of $\mathrm{R} 1 / \mathrm{R} 2$ resections was found for LACC-MV compared with LACCnoMV $(P<.001)$, and in the elective setting only $(P<.001)$. R0 resection proportions were significantly higher in the elective setting compared with the emergency and urgent settings for both LACC-noMV (93\% vs $87 \% ; P<.001)$ and LACC-MV ( $90 \%$ vs $81 \%$; $<.001)$. In the LACCnoMV group, the $\mathrm{RO}$ resection proportion was significantly lower in converted procedures than in laparoscopically completed resections (89\% vs $96 \% ; P<.001)$, although similar R0 resection proportions were found in the LACC-MV group ( $90 \%$ after conversion vs $93 \%$ after laparoscopy). The RO resection proportions after any form of neoadjuvant treatment did not significantly differ from the overall groups.

Table 4 displays data on the postoperative course. The length of stay was longest for the LACC-MV subgroup. Additionally, complications occurred most often in the LACC-MV group. The 30-day/ in-hospital mortality rate was significantly higher for LACC compared with non-LACC (5.8\% vs 3.6\%; $P<.001)$ with multivisceral resection showing no significant impact $P=.606)$ in patients with LACC (Table 4).

\section{Table 2. Guideline Adherence}

\begin{tabular}{|c|c|c|c|c|}
\hline & \multicolumn{2}{|c|}{ LACC-noMV } & \multicolumn{2}{|c|}{ LACC-MV } \\
\hline & $\mathbf{n}$ & $\%$ & $\mathbf{n}$ & $\%$ \\
\hline \multicolumn{5}{|c|}{ Elective, emergency, and urgent procedures } \\
\hline \multicolumn{5}{|l|}{ Preoperative imaging ${ }^{a}$} \\
\hline \multicolumn{5}{|l|}{ Abdomen } \\
\hline None & 93 & $2.8 \%$ & 18 & $1.2 \%$ \\
\hline Ultrasound & 184 & $5.6 \%$ & 61 & $3.9 \%$ \\
\hline $\mathrm{CT}$ & 2,912 & $89 \%$ & 1,406 & $91 \%$ \\
\hline MRI-liver ${ }^{b}$ & 31 & $0.9 \%$ & 15 & $1.0 \%$ \\
\hline $\mathrm{PET} / \mathrm{CT}^{\mathrm{b}}$ & 63 & $1.9 \%$ & 47 & $3.0 \%$ \\
\hline \multicolumn{5}{|l|}{ Thorax } \\
\hline None & 305 & $9.1 \%$ & 86 & $5.5 \%$ \\
\hline Chest x-ray & 2,292 & $68 \%$ & 1,062 & $68 \%$ \\
\hline CT & 694 & $21 \%$ & 373 & $24 \%$ \\
\hline $\mathrm{PET} / \mathrm{CT}^{\mathrm{b}}$ & 65 & $1.9 \%$ & 47 & $3.0 \%$ \\
\hline \multicolumn{5}{|l|}{ Elective procedures only } \\
\hline \multicolumn{5}{|l|}{ Preoperative imaging ${ }^{a}$} \\
\hline \multicolumn{5}{|l|}{ Abdomen } \\
\hline None & 18 & $0.8 \%$ & 3 & $0.3 \%$ \\
\hline Ultrasound & 128 & $5.8 \%$ & 44 & $4.0 \%$ \\
\hline CT & 1,982 & $90 \%$ & 993 & $91 \%$ \\
\hline MRI-liver ${ }^{\mathrm{b}}$ & 26 & $1.2 \%$ & 11 & $1.0 \%$ \\
\hline $\mathrm{PET} / \mathrm{CT}^{\mathrm{b}}$ & 57 & $2.6 \%$ & 40 & $3.7 \%$ \\
\hline \multicolumn{5}{|l|}{ Thorax } \\
\hline None & 79 & $3.5 \%$ & 30 & $2.7 \%$ \\
\hline Chest x-ray & 1,561 & $70 \%$ & 740 & $67 \%$ \\
\hline CT & 538 & $24 \%$ & 294 & $27 \%$ \\
\hline PET-CTa & 58 & $2.6 \%$ & 39 & $3.5 \%$ \\
\hline MDT & 1,784 & $80 \%$ & 919 & $82 \%$ \\
\hline \multicolumn{5}{|l|}{ Elective procedures, cT4 } \\
\hline \multicolumn{5}{|l|}{ Neoadjuvant therapies } \\
\hline Chemotherapy & 2 & $1.1 \%$ & 12 & $6.7 \%$ \\
\hline ChemoRT & 5 & $2.9 \%$ & 17 & $9.5 \%$ \\
\hline
\end{tabular}

Abbreviations: chemoRT, chemoradiotherapy; LACC, locally advanced colon cancer; MDT, multidisciplinary team discussion; MV, multivisceral resection required; noMV, no multivisceral resection required. an the Dutch Surgical Colorectal Audit database, preoperative imaging is registered for the abdomen and thorax separately. The imaging modality is further specified for both compartments, and only the one with highest accuracy is registered in case of multiple modalities. ${ }^{b}$ Analyzed as fulfilling guideline recommendation for preoperative imaging. 
Klaver et al

Table 3. Radicality of Resection

\begin{tabular}{|c|c|c|c|c|c|c|c|}
\hline \multirow{4}{*}{$\begin{array}{r}\frac{}{\text { Overall }} \\
\text { RO }\end{array}$} & \multicolumn{2}{|c|}{ non-LACC } & \multicolumn{2}{|c|}{ LACC-noMV } & \multicolumn{2}{|c|}{ LACC-MV } & \multirow[b]{2}{*}{$\chi^{2}$} \\
\hline & \multirow[t]{2}{*}{$\mathrm{n}$} & \multirow[t]{2}{*}{$\%$} & \multirow[t]{2}{*}{$\mathrm{n}$} & \multirow[t]{2}{*}{$\%$} & \multirow[t]{2}{*}{$\mathrm{n}$} & \multirow[t]{2}{*}{$\%$} & \\
\hline & & & & & & & \\
\hline & 28,605 & $99 \%$ & 2,981 & $91 \%$ & 1,363 & $87 \%$ & $<.001$ \\
\hline R1 & 222 & $0.8 \%$ & 190 & $5.8 \%$ & 135 & $8.7 \%$ & \\
\hline $\mathrm{R} 2$ & 51 & $0.2 \%$ & 93 & $2.8 \%$ & 62 & $4.0 \%$ & \\
\hline \multicolumn{8}{|c|}{ Elective procedures } \\
\hline RO & 24,618 & $99 \%$ & 2,040 & $93 \%$ & 990 & $90 \%$ & $<.001$ \\
\hline R1 & 146 & $0.6 \%$ & 95 & $4.3 \%$ & 79 & $7.2 \%$ & \\
\hline $\mathrm{R} 2$ & 32 & $0.1 \%$ & 50 & $2.3 \%$ & 29 & $2.6 \%$ & \\
\hline \multicolumn{8}{|c|}{ Emergency/Urgent procedures } \\
\hline Ro & 3,949 & $98 \%$ & 938 & $87 \%$ & 373 & $81 \%$ & $<.001$ \\
\hline $\mathrm{R} 1$ & 72 & $1.8 \%$ & 95 & $8.8 \%$ & 56 & $12 \%$ & \\
\hline $\mathrm{R} 2$ & 18 & $0.4 \%$ & 43 & $4.0 \%$ & 33 & $7.1 \%$ & \\
\hline \multicolumn{8}{|l|}{ Open } \\
\hline Ro & 13,453 & $99 \%$ & 1,855 & $89 \%$ & 1,049 & $86 \%$ & $<.001$ \\
\hline $\mathrm{R} 1$ & 132 & $1.0 \%$ & 142 & $6.8 \%$ & 111 & $9.1 \%$ & \\
\hline $\mathrm{R} 2$ & 32 & $0.2 \%$ & 78 & $3.8 \%$ & 57 & $4.7 \%$ & \\
\hline \multicolumn{8}{|c|}{ Laparoscopic } \\
\hline Ro & 13,257 & $99 \%$ & 926 & $96 \%$ & 153 & $93 \%$ & $<.001$ \\
\hline $\mathrm{R} 1$ & 65 & $0.5 \%$ & 31 & $3.2 \%$ & 10 & $6.1 \%$ & \\
\hline $\mathrm{R} 2$ & 12 & $0.1 \%$ & 7 & $0.7 \%$ & 1 & $0.6 \%$ & \\
\hline \multicolumn{8}{|c|}{ Laparoscopic - converted } \\
\hline Ro & 1,895 & $98 \%$ & 200 & $89 \%$ & 161 & $90 \%$ & $<.001$ \\
\hline R1 & 25 & $1.3 \%$ & 17 & $7.6 \%$ & 14 & $7.8 \%$ & \\
\hline $\mathrm{R} 2$ & 7 & $0.4 \%$ & 8 & $3.6 \%$ & 4 & $2.2 \%$ & \\
\hline \multicolumn{8}{|c|}{ Neoadjuvant chemotherapy ${ }^{a}$} \\
\hline Ro & & & 22 & $85 \%$ & 47 & $92 \%$ & $.429^{b}$ \\
\hline R1 & & & 2 & $7.7 \%$ & 3 & $5.9 \%$ & \\
\hline $\mathrm{R} 2$ & & & 2 & $7.7 \%$ & 1 & $2.0 \%$ & \\
\hline \multicolumn{8}{|c|}{ Neoadjuvant (chemo) RT ${ }^{\mathrm{a}}$} \\
\hline Ro & & & 9 & $90 \%$ & 40 & $83 \%$ & $.321^{\mathrm{b}}$ \\
\hline R1 & & & 0 & $0.0 \%$ & 6 & $13 \%$ & \\
\hline R2 & & & 1 & $10 \%$ & 2 & $4.2 \%$ & \\
\hline
\end{tabular}

Abbreviations: chemoRT, chemoradiotherapy; LACC, locally advanced colon cancer; MV, multivisceral resection required; noMV, no multivisceral resection required; R0, complete tumour resection with all margins histologically uninvolved; R1 incomplete resection with microscopic surgical resection margin involvement; $\mathrm{R} 2$, incomplete tumour resection with gross residual tumour that was not resected.

anly elective procedures included.

${ }^{\mathrm{b}}$ Analyzed using Fisher exact test.

Patients with LACC-MV were treated in all 92 hospitals. Based on the number of these patients that were treated, the hospitals were subdivided into lowvolume ( $\leq 5$ procedures annually) and high-volume ( $>5$ procedures annually). There were 82 lowvolume hospitals (median volume, 2.3; range, 0.25.0) and 10 high-volume hospitals (median volume, 6.9; range, 5.2-8.2). The $\mathrm{RO}$ resection proportion was $86 \%$ in low-volume hospitals compared with 91\% in high-volume hospitals $(P=.024)$.

When examining the development of the quality of surgical care throughout the years, a significantly positive trend in completeness of resection, postoperative complicated course, and 30-day/in-hospital mortality could be observed in the non-LACC and LACC-noMV groups. These improvements were less clear (and nonsignificant) in the LACC-MV group (Figure 2).

\section{Discussion}

This population study reports on clinicopathologic characteristics, treatment strategy, and short-term outcomes after resection of MO LACC in 4,980 patients, who composed $13 \%$ of the registered patients who underwent resection for colon cancer during a 
T4 Colon Cancer: Current Practice

\begin{tabular}{|c|c|c|c|c|c|c|c|}
\hline & \multicolumn{2}{|c|}{ non-LACC } & \multicolumn{2}{|c|}{ LACC-noMV } & \multicolumn{2}{|c|}{ LACC-MV } & \multirow[b]{2}{*}{$\chi^{2}$} \\
\hline & $\mathbf{n}$ & $\%$ & $\mathbf{n}$ & $\%$ & $\mathbf{n}$ & $\%$ & \\
\hline $\begin{array}{l}\text { Median length of stay (IQR), } \\
\text { days }\end{array}$ & $7(5-11)$ & & $8(5-14)$ & & $10(7-16)$ & & $<.001^{\mathrm{a}}$ \\
\hline Complicated course ${ }^{b}$ & 5,509 & $17 \%$ & 837 & $25 \%$ & 460 & $29 \%$ & $<.001$ \\
\hline Nonsurgical complications & 3,859 & $13 \%$ & 561 & $17 \%$ & 254 & $16 \%$ & $<.001$ \\
\hline Surgical complications & 3,698 & $13 \%$ & 494 & $15 \%$ & 290 & $18 \%$ & $<.001$ \\
\hline Surgical reintervention & 2,816 & $9.5 \%$ & 355 & $11 \%$ & 185 & $12 \%$ & .007 \\
\hline Radiologic reintervention & 270 & $0.9 \%$ & 47 & $1.4 \%$ & 40 & $2.5 \%$ & $<.001$ \\
\hline Mortality ${ }^{c}$ & 1,070 & $3.6 \%$ & 201 & $6.0 \%$ & 86 & $5.4 \%$ & $<.001$ \\
\hline
\end{tabular}

Abbreviations: IQR, interquartile range; LACC, locally advanced colon cancer; MV, multivisceral resection required; noMV, no multivisceral resection required.

aAnalyzed using Kruskal-Wallis one-way analysis of variance.

${ }^{b}$ Complicated course: postoperative complication leading to a reintervention, hospital stay $>14$ days, or death.

c30-day/in-hospital mortality.

6-year study period in the Netherlands. Only a small proportion of patients with LACC were treated with neoadjuvant chemoRT and/or radiotherapy. The overall R0 resection proportion was $90 \%$ in patients with LACC, with the lowest proportion being $81 \%$ for those who underwent a multivisceral resection in a nonelective setting. Patients with LACC had a slightly worse postoperative outcome compared with non-LACC patients. Short-term outcomes improved over time for the LACC-noMV group, with the R0 resection proportion exceeding $95 \%$. For the LACCMV group, improvement over time was less clear and the $\mathrm{R} 0$ resection proportion in 2014 was $88 \%$.

An R1 resection of a primary colon cancer has a strong and stage-independent negative prognostic impact on the survival and recurrence rate. ${ }^{9}$ In a recent single-institution cohort study, recurrence rates were $56 \%$ and $19 \%$ for R1 and R0 resection, respectively, with corresponding 5-year survival rates of $25 \%$ and $60 \% .{ }^{10}$ Similar to our findings, the risk of incomplete resection was related to the $\mathrm{T}$ stage. $\mathrm{R} 0$ resection proportions were remarkably low: $65 \%$ for $\mathrm{T} 4 \mathrm{a}$ and $50 \%$ for $\mathrm{T} 4 \mathrm{~b}$. Another population-based study reported a $75 \% \mathrm{R} 0$ resection proportion in 861 patients with $\mathrm{T} 4 \mathrm{a}$ stage colon cancer. ${ }^{11}$ These data and our findings suggest that there is room for improvement in surgery for LACC. This will have a positive impact on prognosis given its independent association with recurrence and survival. Furthermore, the $5.8 \%$ postoperative mortality rate for LACC also suggests room for improvement. This mortality rate is comparable to that seen in published series on LACC $(3.3 \%$ $8.9 \%) .{ }^{12-14}$ However, this is a population-based study of unselected patients, including those treated in emergency surgery and nonexpert centers. The volume-outcome relationship in the present analysis suggests the potential benefit of further specialization and centralization of care in high-volume centers. The small differences in absolute numbers of procedures between low- and high-volume hospitals (2.3 vs 6.9, respectively), as well as the relatively low median volume in the high-volume group (6.9), show that LACC surgery has not yet been centralized in the Netherlands. Further improvement might be expected when annual volumes exceed 15 to $20 .^{15}$ The low hospital volumes for LACC-MV might also explain the absence of improvement over time for LACC-MV. Furthermore, lower RO resection proportions in the emergency and urgent settings suggest a potential role for bridging strategies, such as a decompressing stoma. This would enable optimal staging, potential neoadjuvant treatment, and elective surgery by an optimal surgical team.

A multivisceral resection is essential to achieve an $\mathrm{RO}$ resection in $\mathrm{pT} 4 \mathrm{~b}$ stage colon cancer and has been associated with improved outcomes at the population level. ${ }^{16}$ However, preoperative and intraoperative assessment of organ involvement is often inaccurate because of the difficulty in distinguishing between true tumor invasion and inflammatory adhesions. ${ }^{17,18}$ Reported true pT4 rates 
in multivisceral resections were $55 \%, 36 \%$, and $34 \%$ in 3 studies, respectively. ${ }^{13,19,20}$ Therefore, multivisceral resection often turns out to be overtreatment. This is a clinically relevant problem because of the increased morbidity rates, as shown by our results and those of others. ${ }^{14,18}$ Despite its drawbacks, a multivisceral resection seems to be preferred over a less radical approach in clinically adherent tumors with uncertainty regarding the extent of malignant invasion, bearing in mind the negative prognostic impact of an incomplete resection. ${ }^{21-23}$

In addition to extensive surgery, neoadjuvant therapies could optimize R0 resection proportions in LACC. ${ }^{24,25}$ In contrast to other types of gastrointestinal cancer, administration of neoadjuvant therapy in colon cancer remains uncommon. ${ }^{11,14,26,27}$ Incidental use of a variety of neoadjuvant therapy schedules has been described. In the phase II FOxTROT trial, ${ }^{6} 150$ patients with LACC were randomized (2:1) between an experimental arm with preoperative chemotherapy (FOLFOX) and a second randomization in RAS wildtype for an anti-EGFR antibody, and a control arm with routine adjuvant chemotherapy only. Preoperative systemic therapy was shown to reduce tumor size and resulted in a significant improvement of $\mathrm{R} 0$ resection proportion ( $96 \%$ vs $80 \%$ ). The need for emergency or urgent surgery, complication rate, and toxicity were comparable across both groups. These findings were confirmed in another phase II study including 22 patients, ${ }^{28}$ and the PRODIGE 22-ECKINOXE trial with a similar design is currently recruiting. ${ }^{29}$ In the present study, neoadjuvant therapy was not associated with a higher percentage of $\mathrm{R} 0$ resections. This may be the result of both small sample size $(n=77)$ and allocation bias, because the most advanced tumors were probably allocated to neoadjuvant therapy.

Because of concerns regarding radiation toxicity, mainly to the small bowel, the use of chemoRT for LACC remains controversial. ${ }^{30}$ Results of one study in which 33 patients were retrospectively analyzed suggested that neoadjuvant chemoRT combined with en bloc multivisceral resection results in high RO resection proportions and excellent local control, with acceptable morbidity and mortality. ${ }^{17}$ In $64 \%$ of these patients, the T4 tumor was located in the sigmoid; it was also the main tumor location (68\%) in patients who received neoadjuvant chemoRT in the present study.

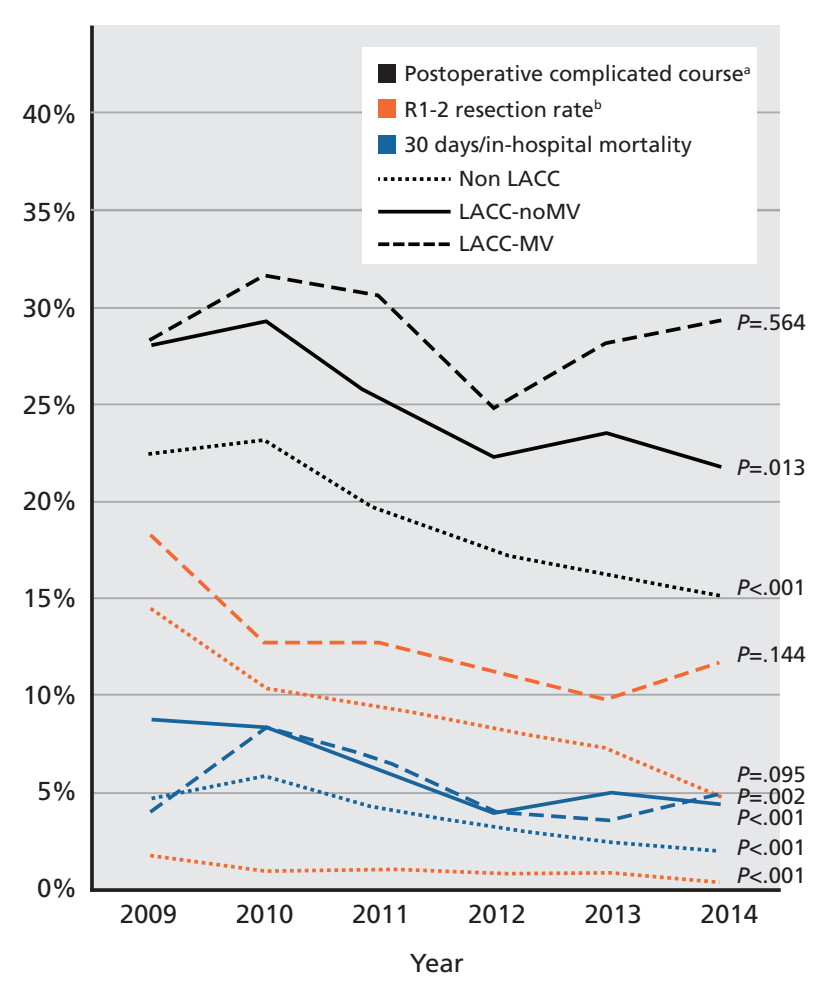

Figure 2. Development of the quality of surgical care for LACC. Abbreviations: LACC, locally advanced colon cancer; MV, multivisceral resection required; noMV, no multivisceral resection required; non-LACC, all other colon cancer resections.

apostoperative complicated course: postoperative complication leading to a re-intervention, hospital stay $>14$ days, or death.

bR1, incomplete resection with microscopic surgical resection margin involvement; R2, incomplete tumour resection with gross residual tumour that was not resected.

Decisions on neoadjuvant therapy strategies should be based on preoperative imaging, but the accuracy is limited and overstaging rates of up to $50 \%$ have been described. ${ }^{13,31}$ In this study, a comparable discrepancy between cT4 and pT4 was found; in 833 of the patients with pT4 tumors, clinical T stage was registered, with $61 \%$ being classified as cT1-3. Only $57 \%$ of the 578 cT4 tumors were classified as pT4 tumors. Despite its limited accuracy, preoperative imaging seems to be essential when considering neoadjuvant treatment and surgical planning. Therefore, further improvement can be expected from optimal guideline adherence with respect to preoperative imaging and MDT discussion.

LACC is often considered a contraindication for laparoscopic surgery because of oncologic concerns. In this series, laparoscopic surgery was performed in $31 \%$ of LACC patients overall and $21 \%$ of LACC$\mathrm{MV}$, with conversion rates of up to $52 \%$. Conversion 
did not lower the $\mathrm{R} 0$ resection proportion in patients with LACC-MV, which suggests that it can be considered safe to initiate surgery laparoscopically. In contrast, conversion did result in lower $\mathrm{R} 0$ resection proportions in the LACC-noMV group. The latter finding is remarkable and was not confirmed in the literature. Several nonrandomized comparative studies have been published on laparoscopy in LACC. ${ }^{11,14,26,27,32,33}$ The laparoscopic group often had favorable baseline characteristics regarding factors such as previous abdominal surgery and emergency setting. Additionally, resections were less often multivisceral. Conversion rates ranged between $7 \%$ and $24 \%$, and $\mathrm{R} 0$ resection proportions were mostly similar to those of the open surgery groups. These data are most likely skewed by allocation bias. Increasing the rate of laparoscopic surgery for LACC might contribute to a lower morbidity rate, but this may never jeopardize oncologic safety.

This is a large population-based cohort study, which provides the best available evidence of the nationwide current clinical practice regarding LACC. However, several limitations of this design should be kept in mind. The availability of data is dependent on the self-reported data from the DSCA database, which is subject to registration bias and incomplete data registration. Nonetheless, in order to show accuracy and completeness of data, these were validated on a yearly basis using the Dutch National Cancer Registry. ${ }^{7}$ Furthermore, the variable set is chosen for the purpose of clinical auditing, and several variables relevant to the aim of this study, such as subdivision in T4a/b subgroups and organ involvement based on pathology reports, are lacking. Additionally, the clinical $\mathrm{T}$ stage was unknown in a substantial number of patients, resulting in a small sample size of clinical T4 tumors, which is the relevant group to assess for neoadjuvant strategies. Furthermore, differences in patient and tumor characteristics between subgroups should be recognized when comparing outcome variables between the relevant subgroups.

\section{Conclusions}

Among patients who undergo surgery for LACC there is a lower $\mathrm{RO}$ resection proportion and they are at higher risk of postoperative complications and mortality compared with patients who receive surgery for less invasive colon cancer. Neoadjuvant therapy for colon cancer is still rarely applied in the Netherlands and prospective randomized studies must be awaited in order to confirm the observation of more radical resections in phase II studies. Considering the relatively low $\mathrm{RO}$ resection proportion, there is an opportunity for improvement. This may be achieved by optimizing preoperative imaging, the application of neoadjuvant therapy schedules, and centralization and specialization.

\section{Acknowledgments}

The authors would like to thank Daphne Dijkman for the language editing.

\section{References}

1. Ferlay J, Soerjomataram I, Dikshit R, et al. Cancer incidence and mortality worldwide: sources, methods and major patterns in GLOBOCAN 2012. Int J Cancer 2014;136:E359-386.

2. Izbicki JR, Hosch SB, Knoefel WT, et al. Extended resections are beneficial for patients with locally advanced colorectal cancer. Dis Colon Rectum 1995;38:1251-1256.

3. Saha AK, Smith KJE, Sue-Ling H, et al. Prognostic factors for survival after curative resection of Dukes' B colonic cancer. Colorectal Dis 2011;13:1390-1394

4. Edge S, Byrd DR, Compton CC, et al, eds. AJCC Cancer Staging Manual, 7th ed. New York, NY: Springer; 2010.

5. Larkin JO, O'Connell PR. Multivisceral resection for T4 or recurrent colorectal cancer. Dig Dis 2012;30(Suppl 2):96-101.

6. Foxtrot Collaborative Group. Feasibility of preoperative chemotherapy for locally advanced, operable colon cancer: the pilot phase of a randomised controlled trial. Lancet Oncol 2012;13:1152-1160.

7. van Leersum NJ, Snijders HS, Wouters MW, et al. Evaluating national practice of preoperative radiotherapy for rectal cancer based on clinical auditing. Eur J Surg Oncol 2013;39:1000-1006.
8. Kolfschoten NE, Marang van de Mheen PJ, Gooiker GA, et al. Variation in case-mix between hospitals treating colorectal cancer patients in the Netherlands. Eur J Surg Oncol 2011;37:956-963.

9. Amri R, Bordeianou LG, Sylla P, Berger DL. Association of radial margin positivity with colon cancer. JAMA Surg 2015;150:890-898.

10. Khan MA, Hakeem AR, Scott N, Saunders RN. Significance of R1 resection margin in colon cancer resections in the modern era. Colorectal Dis 2015;17:943-953.

11. Elnahas A, Sunil S, Jackson TD, et al. Laparoscopic versus open surgery for T4 colon cancer: evaluation of margin status. Surg Endosc 2016;30:14911496.

12. Campos FG, Calijuri-Hamra MC, Imperiale AR, et al. Locally advanced colorectal cancer: results of surgical treatment and prognostic factors. Arq Gastroenterol 48:270-275.

13. Gezen C, Kement M, Altuntas YE, et al. Results after multivisceral resections of locally advanced colorectal cancers: an analysis on clinical and pathological t4 tumors. World J Surg Oncol 2012;10:39.

14. Hoffmann M, Phillips C, Oevermann E, et al. Multivisceral and standard resections in colorectal cancer. Langenbecks Arch Surg 2012;397:75-84.

15. Gietelink L, Henneman D, van Leersum NJ, et al. The influence of hospital volume on circumferential resection margin involvement: results of the Dutch Surgical Colorectal Audit. Ann Surg 2016;263:745-750. 


\section{Klaver et al}

16. Govindarajan A, Coburn NG, Kiss A, et al. Population-based assessment of the surgical management of locally advanced colorectal cancer. J Natl Cancer Inst 2006;98:1474-1481.

17. Cukier M, Smith AJ, Milot L, et al. Neoadjuvant chemoradiotherapy and multivisceral resection for primary locally advanced adherent colon cancer: a single institution experience. Eur J Surg Oncol 2012;38:677-682.

18. Mohan HM, Evans MD, Larkin JO, et al. Multivisceral resection in colorectal cancer: a systematic review. Ann Surg Oncol 2013;20:2929_ 2936.

19. Gebhardt C, Meyer W, Ruckriegel S, Meier U. Multivisceral resection of advanced colorectal carcinoma. Langenbecks Arch Surg 1999;384:194199.

20. Darakhshan A, Lin BPFC, Chan C, et al. Correlates and outcomes of tumor adherence in resected colonic and rectal cancers. Ann Surg 2008;247:650-658.

21. Cirocchi R, Partelli S, Castellani E, et al. Right hemicolectomy plus pancreaticoduodenectomy vs partial duodenectomy in treatment of locally advanced right colon cancer invading pancreas and/or only duodenum. Surg Oncol 2014;23:92-98.

22. Zhang J, Leng J, Qian H, et al. En bloc pancreaticoduodenectomy and right colectomy in the treatment of locally advanced colon cancer. Dis Colon Rectum 2013;56:874-880.

23. Croner RS, Merkel S, Papadopoulos $\mathrm{T}$, et al. Multivisceral resection for colon carcinoma. Dis Colon Rectum 2009;52:1381-1386.

24. Stojadinovic A, Nissan A, Wainberg Z, et al. Time-dependent trends in lymph node yield and impact on adjuvant therapy decisions in colon cancer surgery: an international multi-institutional study. Ann Surg Oncol 2012;19:4178-4185.
25. Scott N, Jamali A, Verbeke C, et al. Retroperitoneal margin involvement by adenocarcinoma of the caecum and ascending colon: what does it mean? Color Dis 2008;10:289-293.

26. Vignali A, Ghirardelli L, Di Palo S, et al. Laparoscopic treatment of advanced colonic cancer: a case-matched control with open surgery. Color Dis 2013;15:944-948.

27. Shukla PJ, Trencheva K, Merchant C, et al. Laparoscopic resection of T4 colon cancers: is it feasible? Dis Colon Rectum 2015;58:25-31.

28. Arredondo J, Pastor C, Baixauli J, et al. Preliminary outcome of a treatment strategy based on perioperative chemotherapy and surgery in patients with locally advanced colon cancer. Colorectal Dis 2013;15:552-557.

29. Karoui M, Rullier A, Luciani A, et al. Neoadjuvant FOLFOX 4 versus FOLFOX 4 with cetuximab versus immediate surgery for high-risk stage Il and III colon cancers: a multicentre randomised controlled phase II trial the PRODIGE 22 - ECKINOXE trial. BMC Cancer 2015;15:511.

30. Hallet J, Zih FS, Lemke M, et al. Neo-adjuvant chemoradiotherapy and multivisceral resection to optimize RO resection of locally recurrent adherent colon cancer. Eur J Surg Oncol 2014;40:706-712.

31. van Santvoort HC, Braam HJ, Spekreijse KR, et al. Peritoneal carcinomatosis in $\mathrm{t} 4$ colorectal cancer: occurrence and risk factors. Ann Surg Oncol 2014;21:1686-1691.

32. Huh JW, Kim HR. The feasibility of laparoscopic resection compared to open surgery in clinically suspected T4 colorectal cancer. J Laparoendosc Adv Surg Tech A 2012;22:463-467.

33. Kim IY, Kim BR, Kim YW. The short-term and oncologic outcomes of laparoscopic versus open surgery for T4 colon cancer. Surg Endosc 2016;30:1508-1518.

\section{See JNCCN.org for supplemental online content.}

